\title{
PERFIL EPIDEMIOLÓGICO DEL SERVICIO DE URGENCIAS SEGÚN MOTIVO DE CONSULTA HOSPITAL DE SAN JOSÉ BOGOTÁ DC, SEPTIEMBRE 2011 A FEBRERO 2012
}

Erich Steve Picón Silva MD*, Julián Hernando Salgado Hernández MD*, Miguel Angel Saavedra Ortiz MD**

\section{Resumen}

Introducción: el servicio de urgencias del Hospital de San José de Bogotá DC adelanta la implementación de un programa de registros clínicos con fines estadísticos y propósitos de investigación. Objetivo: establecer el perfil epidemiológico del servicio de urgencias de adultos con base en la información del software e-salud en un período de seis meses. Métodos: se obtuvieron 18.519 registros de septiembre 2011 a febrero 2012. Las variables consideradas fueron edad, género, responsable del pago de la atención, clasificación triage, tiempo de espera para la atención y motivo de consulta; los datos fueron procesados con frecuencias, medidas de tendencia central y dispersión en Stata 10. Resultados: la población atendida en su mayoría fue menor de 50 años $(65 \%), 52.6 \%$ fueron hombres, la edad promedio 43 años, el principal pagador corresponde a la aseguradora (EPS 90.3\%), los motivos de consulta más frecuentes fueron dolor abdominal (61.2\%), trauma $(14.2 \%)$ y cefalea $(10,2 \%)$. El tiempo de espera para la atención fue menor de quince minutos en el $85 \%$ de los casos. Conclusión: no existieron diferencias entre el perfil epidemiológico obtenido con la información arrojada por el sistema de registro $e$-Salud y el previo del personal de facturación. Los hallazgos sugieren ajustar y ampliar las guías trazadoras del servicio.

Palabras clave: perfil epidemiológico, triage, motivo de consulta, servicio de urgencias.

\section{EPIDEMIOLOGICAL PROFILE OF THE EMERGENCY DEPARTMENT ACCORDING TO REASONS FOR CONSULTATION - HOSPITAL DE SAN JOSÉ BOGOTÁ DC - SEPTEMBER 2011 TO FEBRUARY 2012}

\section{Abstract}

Introduction: the emergency department at Hospital de San José, Bogotá DC is currently implementing a clinical information recording system for statistical and research purposes. Objective: to establish the adult ER epidemiological profile based on data obtained with the $e$-Salud software during a six-month period. Methods: we obtained 18,519 records between September 2011 and February 2012. Variables analyzed included: age, gender, payer of healthcare services, triage classification, wait time and reason for consultation; Stata 10 was utilized for data analysis using frequencies

Fecha recibido: julio 4 de 2012 - Fecha aceptado: febrero 15 de 2013

* Residente III de Medicina de Urgencias, Fundación Universitaria de Cienciasde la Salud. Bogotá DC, Colombia.

** Especialista en Medicina de Urgencias y Cirugía Vascular Periférica Coordinador del servicio de urgencias del Hospital de San José y del programa de Medicina de Urgencias, Instructor Asistente, Fundación Universitaria de Ciencias de la Salud, Bogotá DC, Colombia.

*** Merideidy Plazas, asesora metodológica. Profesora Asociada de Epidemiología, División de Investigaciones, Fundación Universitaria de Ciencias de la Salud, Bogotá DC, Colombia. 
and central and dispersion tendency measures. Results: patients seen were mostly under age $50(65 \%), 52.6 \%$ were males, average age was 43 years, the main payer was patient's insurance company (EPS 90.3\%), the main reasons for consultation were abdominal pain $(61.2 \%)$, trauma $(14.2 \%)$ and headache $(10.2 \%)$. Wait time was less than 15 minutes in $85 \%$ of cases. Conclusion: no differences were found between the epidemiological profile obtained with the $e$-Salud software and the information garnered from the ER invoicing staff. Findings suggest that ER care tracking guidelines must be adjusted and enlarged.

Key words: epidemiological profile, TRIAGE, reason for consultation, emergency department

\section{Introducción}

El Hospital de San José de Bogotá DC es una institución con más de 100 años de historia que lo ha convertido en centro de referencia no solo en la ciudad sino a nivel nacional, dada su capacidad de resolución y alto nivel científico. Ha recibido estudiantes de pre y posgrado de las principales facultades de medicina del país y en la actualidad es uno de los sitios de práctica más importantes de los alumnos de la Fundación Universitaria de Ciencias de la Salud.

El servicio de urgencias tiene la capacidad de atender siete pacientes de manera simultánea, en consultorios individuales que cumplen con los requisitos de habilitación. Hay una unidad de reanimación con cinco camas, un quirófano para intervenciones quirúrgicas de emergencia, sala para procedimientos menores y áreas de manejo de pacientes con problemas respiratorios y para rehidratación. En el segundo piso se encuentra el área de observación que cuenta con 28 camillas para estancia menor a seis horas. El hospital oferta todas las especialidades de III y IV niveles y el servicio dispone de un grupo de especialistas en medicina de urgencias y residentes de esta especialidad durante las 24 horas diarias toda la semana. Se atienden pacientes mayores de 14 años y 6 meses y mujeres que no tengan como motivo de consulta embarazo confirmado o patologías relacionadas con este.

Desde el año 2002 las directivas del hospital decidieron la implementación de un sistema de registro digital de la historia clínica, como parte del proceso de actualización constante que ha caracterizado a la institución y además para responder a las necesidades incluidas en el sistema único de garantía de la calidad y los manuales de acreditación para instituciones de salud. Con tal fin se creo un grupo multidisciplinario conformado por personal administrativo y asistencial, que se denominó $e$-salud..$^{1-3}$

Los miembros del equipo adelantaron un proceso de selección con participación de varios proveedores nacionales y extranjeros, pero al no encontrar un sistema que se adaptara a la totalidad de las características y requerimientos de los diferentes usuarios y con el apoyo de las directivas, decidieron crear un sistema de registros clínicos propio que cuenta con diferentes ambientes, con el fin de integrar la información administrativa y asistencial, además de interactuar con el sistema de facturación Hipócrates que había sido adquirido varios años antes.

En la actualidad este grupo de desarrollo ha entregado una herramienta con el programa (software) bajo el nombre $e$-salud, que se encuentra en implementación al interior del hospital. Al momento de escribir el presente artículo se encuentran sistematizados los registros de la consulta externa, salas de cirugías, además de la atención en el servicio de urgencias ginecológicas, que corresponde a una cobertura del $40 \%$ de la institución.

Desde septiembre de 2011 se inició la implementación de dicho software en el servicio de urgencias del Hospital de San José. En la actualidad se desarrolla la primera fase compuesta por dos módulos: el primero es el ingreso diligenciado por la enfermera orientadora. Contiene los datos de identificación, información demográfica y administrativa del paciente así como el registro inicial de las constantes vitales. El segundo módulo corresponde al de clasificación inicial 
o triage, bajo la responsabilidad del personal médico. En este se analizan las constantes vitales, el motivo de consulta informado por el paciente y se asigna la clasificación de gravedad con base en la escala triage, que para la institución se encuentra dividida en cuatro niveles siendo el I urgencia vital, el II urgencia, el III prioritario y el IV diferible a consulta programada, tomando como referencia los protocolos establecidos por la Universidad de Manchester en Inglaterra y adoptados como estándar en la mayoría de servicios de urgencia con algunas variaciones propias. ${ }^{4-6}$

La clasificación inicial con los estándares relacionados establece que el nivel I requiere atención inmediata, el II antes de quince minutos y los III y IV pueden ser derivados para atención prioritaria fuera del servicio de urgencias. ${ }^{7}$ La iniciación de la sistematización de los registros clínicos se convirtió en una oportunidad para conocer el perfil epidemiológico del servicio con base en los datos registrados por el profesional encargado de la atención, pues antes de la implementación del software los registros estadísticos se obtenían mediante la interpretación que hacía el funcionario encargado de facturación y de los datos clínicos anotados de manera manuscrita por el médico tratante.

Con la puesta en marcha del sistema de información se minimiza la intervención de intermediarios y se espera que con los datos obtenidos se mejore de manera considerable la calidad de la información que es remitida a los organismos de control y vigilancia según la normatividad actual. ${ }^{1}$ Para la elaboración del perfil epidemiológico es necesario tener claridad cuál es la definición y qué se busca con su elaboración. Recordemos que este corresponde a la forma como expresamos la carga de la enfermedad en una población determinada, es decir el estado de salud de los miembros que la componen. Para esta descripción poblacional es necesario conocer algunas de las características que la definen como son la edad, género, morbilidad y estado socioeconómico. ${ }^{8,9}$

La implementación de sistemas de registros clínicos integra los datos demográficos, administrativos y asistenciales de los pacientes atendidos, formando una gran base de datos que al ser analizada permitirá acceder a los datos necesarios para la elaboración del perfil epidemiológico del servicio, en donde se encuentran las características propias de la población objeto, su morbilidad y la oportunidad de la atención prestada. La información obtenida se convierte en una herramienta importante no solo desde el punto de vista administrativo sino asistencial, encaminada a la toma de decisiones y a la creación de espacios de investigación. ${ }^{10}$

\section{Métodos}

Estudio descriptivo retrospectivo que incluyó los pacientes registrados en el software e-salud en el período comprendido entre el 1 de septiembre de 2011 y el 28 de febrero de 2012, mayores de 14 años 6 meses. Se excluyeron mujeres con sintomatología derivada de embarazo confirmado en urgencias, ya que estas son atendidas en forma directa en el servicio de ginecología. Las variables estudiadas fueron edad (años cumplidos) y género, además de algunas administrativas como las empresas administradoras del plan de beneficios responsables del pago de la atención, las cuales se agruparon según sus características en EPS, EPS-S, ARP, SOAT, fiduciarias, IPS, particulares y otros (Glosario).

Otras variables incluidas fueron la clasificación del triage, con el tiempo de espera para la atención según el nivel asignado por el médico responsable y el motivo de consulta que se define como el problema principal o el síntoma fundamental que informa el paciente. Para establecer la frecuencia de presentación se agruparon por sistemas afectados. Los datos se obtuvieron del sistema de información de e-salud en formato plano (txt). Las variables cualitativas se analizaron con frecuencias y las cuantitativas por medio de medidas de tendencia central y dispersión en Stata10.

\section{Resultados}

Durante el período evaluado solicitaron atención 20.221 pacientes, los cuales ingresaron al sistema en su 
totalidad. De estos 1.780 fueron excluidos por presentar inconsistencias en la información suministrada. Es decir que se utilizaron el $90.4 \%$ de los datos ingresados al sistema, que corresponden a 18.441 para el análisis. Se atendieron en promedio durante el período evaluado 3.370 consultas al mes y 112 consultas al día. La mayor demanda se identificó de lunes (excepto festivos cuando la demanda disminuye) a sábado en las franjas de 9 am a $12 \mathrm{~m}$ y de 2 a 5 pm. Los meses de menor demanda fueron noviembre y diciembre de $2011 \mathrm{y}$ enero de 2012. El mes de mayor demanda para el período fue febrero 2012 (Tabla 1).

La razón hombre:mujer es de 1,1:1. El 67\% de los registros analizados corresponden a menores de 50 años y $52.6 \%$ son hombres. El grupo de edad en el que se presentó el mayor número de datos fue entre 21 y 30 años con 4.476 registros. La clasificación inicial del triage más frecuente fue II (urgencia) con 12.432 registros y se identificó un pequeño grupo de pacientes que no respondieron al llamado (Tabla 1).

Las entidades promotoras de salud (EPS) son las principales pagadoras de la atención de los usuarios (90.3\%), siendo en orden descendente Salud Total, Saludcoop y Famisanar. El segundo renglón de pagadores corresponde a los particulares con $5.5 \%$ de los registros y el tercero a las aseguradoras del SOAT con un $2.2 \%$ de la facturación del servicio de urgencias (Tabla 1).

El principal motivo de consulta en menores de 50 años fue dolor abdominal con 3.434 registros, $30.7 \%$ en hombres y $24.6 \%$ en mujeres, seguido por trauma con $2.007(16.2 \%)$ y el tercer lugar lo ocupa la cefalea con $1.460(11.8 \%)$ (Tabla 2). Para los mayores de 50 años el diagnóstico que más se presenta corresponde a la ingestión de cuerpos extraños (1.709), las mujeres aportan el $53.6 \%$ y los hombres $46.4 \%$. Los dos siguientes grupos de diagnósticos frecuentes corresponden a sintomatología ocular $12.3 \%$ y dolor abdominal $10.1 \%$ (Tabla 3). El $73 \%$ de los pacientes que solicitan atención al servicio de urgencias en el Hospital de San José reciben su clasificación y valoración inicial dentro de los primeros quince minutos (Tabla 4).

\section{Discusión}

La información obtenida a partir de los datos demográficos mostró un comportamiento similar a lo reportado en la encuesta nacional de demografía y salud 2010, que describe cómo los adultos que consultan con mayor frecuencia a los servicios de salud son menores de 50 años; así mismo hay similitud en el perfil de aseguramiento al régimen contributivo entre la población económica activa, pues los afiliados al régimen contributivo que consultan con mayor frecuencia son los que se encuentran en edad productiva, es decir menores de 50 años con un pico entre los 21 y 30 años. Si bien en el país la distribución de personas afiliadas al sistema general de salud se encuentran divididas de manera homogénea entre usuarios contributivos y subsidiados, los primeros constituyen la mayor parte de los atendidos en el Hospital de San José, lo cual puede estar relacionado con su ubicación cercana a hospitales públicos a donde suelen acudir los del régimen subsidiado del área del centro de la ciudad. ${ }^{11}$ $\mathrm{Al}$ analizar los motivos de consulta se confirmó que el dolor abdominal ocupa el primer lugar, en una distribución homogénea con respecto al género. Por ello, para esta patología el servicio cuenta con una guía actualizada que se encuentra en proceso de evaluación de adherencia.

El segundo motivo de consulta más común es el trauma, donde las mujeres sobrepasan a los hombres casi 3 a 1 , lo que nos podría insinuar un riesgo relacionado con las actividades que las mujeres del área de influencia desempeñan, así como descartar la presencia de violencia intrafamiliar que debe ser objeto de futuras investigaciones.

En cuanto al tiempo de espera, el hospital se encuentra ajustado a los estándares establecidos según la clasificación triage, sin embargo es importante destacar que $1.4 \%$ de los clasificados con triage 1 se reportan con tiempo superior a cinco minutos lo cual se puede relacionar con la gravedad de los síntomas, pues el ingreso al sistema de historias clínicas se hace después de la atención. Esto requiere especial análisis puesto que podría generar factores de confusión o alteraciones en la validación de los estándares de calidad informa- 
Tabla I. Características demográficas de la población atendida en el servicio de urgencias

\section{Variables}

Masculino

Femenino

Total

n (\%)

Población

Pacientes atendidos

Edad

$<15$ años

15 a 20

21 a 30

31 a 40

41 a 50

51 a 60

$9702(52.6)$

61 a 70

$>$ de 70

$43(0.2)$
$875(4.7)$
$2284(12.4)$
$1659(9)$
$1593(8.6)$
$1237(6.7)$
$912(4.9)$
$1099(6)$

$8739(47.3)$

I844I (100)

$33(0.2)$

$76(0.4)$

$702(3.8)$

$1577(8.6)$

$2192(11.9)$

$4476(24.3)$

$1698(9.2)$

$3357(18.2)$

$1355(7.3)$

$2948(16)$

$1003(5.4)$

$2240(12.1)$

$850(4.5)$

$1762(9.6)$

$906(4.9)$

$2005(10.9)$

TRIAGE

\begin{tabular}{|l|r|r|r|}
\hline I & $58(0.3)$ & $93(0.5)$ & I5I (0.8) \\
\hline II & $6340(34.4)$ & $6902(37.4)$ & I2432(67.4) \\
\hline III & $2937(15.9)$ & $2248(12.2)$ & 5 I85(28.I) \\
\hline IV & $330(1.8)$ & $28 I(1.5)$ & $6 I I(3.3)$ \\
\hline no responden & $37(0.2)$ & $25(0.1)$ & $62(0.3)$ \\
\hline
\end{tabular}

\section{Administradora de plan de beneficios}

\begin{tabular}{|l|r|r|r|}
\hline EPS & $8904(48.3)$ & $7761(42.1)$ & $16665(90.4)$ \\
\hline EPS-S & $20(0.1)$ & $45(0.2)$ & $65(0.4)$ \\
\hline SOAT & $163(0.9)$ & $249(1.4)$ & $4 I 1(2.2)$ \\
\hline ARP & $22(0.1)$ & $81(0.4)$ & $103(0.6)$ \\
\hline IPS & $4(0.02)$ & $3(0.02)$ & $7(0.04)$ \\
\hline régimen especial $\dagger$ & $20(0.1)$ & $9(0.05)$ & $29(0.16)$ \\
\hline consorcios $\ddagger$ & $2(0.01)$ & $7(0.04)$ & $9(0.05)$ \\
\hline particular & $496(2.7)$ & $5.34(2.9)$ & $1030(5.6)$ \\
\hline pagador no identificado & $31(0.2)$ & $24(0.1)$ & $56(0.3)$ \\
\hline medicina prepagada & $40(0.2)$ & $26(0.1)$ & $66(0.3)$ \\
\hline Pacientes atendidos por mes & & & $3196(17.3)$ \\
\hline septiembre & $1720(9.3)$ & $1476(8)$ & $2782(15.1)$ \\
\hline octubre & $1462(7.9)$ & $1320(7.2)$ & $2823(15.3)$ \\
\hline noviembre & $1456(7.9)$ & $1367(7.4)$ & $2747(14.9)$ \\
\hline diciembre & $1395(7.6)$ & $1352(7.3)$ & $288 I(15.6)$ \\
\hline enero & $1529(8.3)$ & $1352(7.3)$ & $4012(21.8)$ \\
\hline febrero & $2140(11.6)$ & $1872(10.2)$ & \\
\hline
\end{tabular}

† Profesores, fuerzas armadas, policía, ferrocarriles nacionales; ‡ Fisalud, Fosyga. 


\begin{tabular}{|c|c|c|c|}
\hline$n(\%)$ & Hombres & Mujeres & Total \\
\hline \multicolumn{4}{|l|}{ Diagnóstico } \\
\hline $\begin{array}{l}\text { I. Dolor } \\
\text { abdominal }\end{array}$ & $1.997(57,3)$ & $1.490(42.7)$ & $3.487(28)$ \\
\hline 2. Trauma & $729(36,2)$ & $1.278(63.7)$ & $2.007(16.1)$ \\
\hline 3. Cefalea & $870(59,6)$ & $590(40,4)$ & $1.460(11.7)$ \\
\hline $\begin{array}{l}\text { 4. Cuerpo extraño } \\
\text { esofágico }\end{array}$ & $678(57.8)$ & $494(42.2)$ & $1.172(9.4)$ \\
\hline $\begin{array}{l}\text { 5. Síntomas } \\
\text { oculares }\end{array}$ & $333(33,7)$ & $656(66.3)$ & $989(8)$ \\
\hline 6. Dolor lumbar & $566(61.7)$ & $352(38,3)$ & $918(7.4)$ \\
\hline $\begin{array}{l}\text { 7. Dolor torácico, } \\
\text { palpitaciones }\end{array}$ & $376(57,7)$ & $276(42.3)$ & $652(5.2)$ \\
\hline 8. Disnea y ahogo & $328(56,6)$ & $25 I(43.4)$ & $579(4.7)$ \\
\hline $\begin{array}{l}\text { 9. Masas y } \\
\text { abultamientos }\end{array}$ & $252(54.3)$ & $212(45.7)$ & $464(3.7)$ \\
\hline 10. Postoperatorio & $164(46.5)$ & $189(53.5)$ & $353(2.8)$ \\
\hline II. Otros & $161(45.6)$ & $192(54,4)$ & $353(2.8)$ \\
\hline Total & $6.454(51.9)$ & $5.980(48.1)$ & $12.434(67.4)$ \\
\hline
\end{tabular}

\begin{tabular}{|c|c|c|c|}
\hline \multicolumn{4}{|c|}{$\begin{array}{l}\text { Tabla 3. Principales motivos de con } \\
\text { pacientes mayores de } 50 \text { años }\end{array}$} \\
\hline$n(\%)$ & Hombres & Mujeres & Total \\
\hline \multicolumn{4}{|l|}{ Diagnóstico } \\
\hline $\begin{array}{l}\text { I. Cuerpo extraño } \\
\text { en esófago }\end{array}$ & $793(46.4)$ & $916(53.6)$ & I.709 (28.5) \\
\hline $\begin{array}{l}\text { 2. Sintomatología } \\
\text { ocular }\end{array}$ & $40 \mathrm{I}(54.3)$ & $338(45.7)$ & 739 (12.3) \\
\hline $\begin{array}{l}\text { 3. Dolor } \\
\text { abdominal }\end{array}$ & $370(60.6)$ & $232(38.5)$ & $602(10)$ \\
\hline $\begin{array}{l}\text { 4. Disnea, ahogo y } \\
\text { otros }\end{array}$ & $372(63)$ & $218(36.9)$ & $590(9.8)$ \\
\hline 5. Dolor torácico & $273(5 \mathrm{I} .5)$ & $257(48.4)$ & $530(8.8)$ \\
\hline 6. Trauma & $266(62.4)$ & $160(37.5)$ & $426(7.1)$ \\
\hline 7. Dolor de cabeza & $199(47)$ & $224(52.9)$ & $423(7.1)$ \\
\hline 8. Lumbalgia & $218(62.1)$ & $133(37.8)$ & 351 (5.9) \\
\hline $\begin{array}{l}\text { 9. Masas y } \\
\text { abultamientos }\end{array}$ & $\mid 44(6 \mid .2)$ & 91 (38.7) & 235 (3.9) \\
\hline 10. Postoperatorio & $110(50.9)$ & $106(49)$ & $216(3.6)$ \\
\hline 10. Otros & $93(52.2)$ & $84(47.4)$ & $177(3)$ \\
\hline Total & $3.248(54)$ & $2.759(45.9)$ & $5.998(32.5)$ \\
\hline
\end{tabular}

\begin{tabular}{|c|c|c|c|c|c|}
\hline$n(\%)$ & 0 a $5 \mathrm{~min}$. & 6 a $15 \mathrm{~min}$. & 16 a $30 \mathrm{~min}$. & $>30 \mathrm{~min}$. & Total \\
\hline Triage I & $122(1.1)$ & $19(0.7)$ & II (0.5) & $10(0.4)$ & $162(0.9)$ \\
\hline Triage II & $6.793(62,5)$ & $2.621(63.4)$ & $1.516(67.8)$ & I.725 (67.3) & II.655 (63.4) \\
\hline Triage III & $3.728(34.3)$ & $655(25.6)$ & 601 (26.9) & $802(29.5)$ & $5.786(3 \mid .5)$ \\
\hline Triage IV & $221(2)$ & $261(10.2)$ & $109(4.8)$ & $129(4.7)$ & 720 (3.9) \\
\hline
\end{tabular}

dos por la institución. Para evitar esto se recomienda un estudio observacional de tiempos y movimientos de atención.

Cabe destacar que el motivo de consulta ubicado en el décimo lugar corresponde a pacientes con sintomatología relacionada con postoperatorio reciente, pero no se logró identificar si correspondían a procedimientos quirúrgicos de la institución, lo que hace necesario estudios complementarios al respecto.

Existe un grupo de pacientes que no espera la atención médica y se desconoce el motivo de la deserción. Como los pacientes ingresan al sistema pero no son atendidos, ocasionan congestión en el servicio de ur- gencias, afectando los estándares de calidad que rigen el quehacer diario.

La revisión inicial de la base de datos de consulta en el servicio de urgencias arrojó un porcentaje de error en la digitación inferior al 10\%. Pese a que es bajo teniendo en cuenta el tamaño de la base de datos, es necesario realizar actividades encaminadas a la reinducción y retroalimentación de los operadores del sistema con el fin de asegurar la calidad y precisión de la información suministrada.

En el procesamiento de los datos se encontró una limitación adicional en el sistema de historias clínicas al no contar con una herramienta de clasificación por 
síntomas, signos o sistemas que permita agrupar de manera eficiente los motivos de consulta en el servicio de urgencias. Es importante tenerla para conocer en detalle las causas de morbilidad y no generar sesgos de interpretación por parte del personal a cargo de la digitación, que podría ser el sistema de clasificación de enfermedades estandarizado por la organización mundial de la salud CIE-10.

\section{Conclusión}

Desde el punto de vista operativo el servicio de urgencias UR-HSJ cumple con los estándares de calidad propuestos en la reglamentación vigente en el país, los cuales se basan en normas internacionales en lo relativo a los tiempos de espera para la atención según la clasificación inicial triage asignada por el médico responsable.

Si bien el perfil epidemiológico producto de esta investigación es similar al obtenido con base en la metodología manual ya descrita, la importancia de obtener esta información radica en que se descarga al facturador de labores adicionales, lo que debería traducirse en una optimización en los tiempos del proceso administrativo al eliminar el reproceso de la información.

Los datos obtenidos a partir del análisis de la base de datos se convirtió en una herramienta adicional del seguimiento de la implementación del sistema $e$-salud en el servicio de urgencias. Se presenta un resultado favorable en la primera fase y es una invitación para continuarla con los demás módulos de software desarrollados para el registro de la atención.

Por último, los adelantos y avances para la entrada en funcionamiento del módulo de consulta médica así como el de evolución de pacientes ingresados al servicio de urgencias, permitirá desarrollar etapas continuadas de este proyecto. Así se podrá obtener una fotografía del quehacer diario del servicio de urgencias y una amplia perspectiva que se convertirá en una herramienta imprescindible para la toma de decisiones administrativas, asistenciales e investigativas.

\section{Glosario}

EPS: empresa promotora de salud.

EPS-S: empresa promotora de salud, régimen subsidiado.

$A R P$ : aseguradora de riesgos profesionales.

SOAT: seguro obligatorio de accidentes de tránsito.

Fiduciarios: usuario que paga a través de fiducia.

Particulares: usuario que no está afiliado a un sistema de seguridad social o si lo está no desea utilizarlo.

FISALUD: régimen estatal que controla los recursos del SOAT.

FOSYGA: fondo de solidaridad y garantía.

\section{Referencias}

1. Colombia. Ministerio de Salud. Decreto 2309 de 2002 (Octubre 15) por el cual se define el Sistema Obligatorio de Garantía deCalidad de la Atención de Salud del Sistema General de Seguridad Social enSalud. Publicado en Diario Oficial44967, 17 de Octubre de 2002

2. Colombia. Ministerio de la Protección Social. Resolución 001446 de 2006 (mayo 8) por la cual se define el Sistema de Información para laCalidad y se adoptan los indicadores de monitoria del Sistema Obligatorio deGarantía de Calidad de la Atención en Salud.). Publicado en Diario Oficial 46271, 17 de Mayo de 2006.

3. Colombia. Presidencia. Decreto 1011 de 2006 (Abril 3) por el cual se establece el Sistema Obligatorio de Garantíade Calidad de la Atención de Salud del Sistema General de Seguridad Social enSalud. Publicado en Diario Oficial 46230 de 2006.

4. Blázquez Rodríguez MC, Chozas García B, Domínguez Pérez L, Leal de Pedro E Navarro Raya A, Sánchez Juan A. Estudio de las urgencias hospitalarias soporte: Triage Manchester. Servicio de Urgencias R.G. Hospital Universitario 12 de Octubre de Madrid. [monografía en Internet]. Mdrid, España: El Hospital; 2005. [Citado Abril 5 de 2013]. Disponible en: http://www.codem.es/Documentos/ Informaciones/Publico/7e040f14-0bea-421f-b327-440fe67f3617/fcd3a6fdbb72-48b2-85a4-f4fad6bfdef5/664e7c6e-4986-4ceb-98a4-14e2fc7905ba/ estudio_urgencias_hosp_triage_manchester.pdf

5. Soler W, Gómez Muñoz M, Bragulat E, Álvarez A. El triaje: herramienta fundamental en urgencias y emergencias. Anales Sis San Navarra. 2012;33:55-68.

6. Zarante MC, Gómez M. Perfil epidemiológico depacientes que consultaron por intoxicación con psicoactivos a una clínica deMedellín.[Tesis] In. 4 - Feb- 2011; 2012

7. Vallejo GG, Marta Lía V, Nelson Armando A, Liliana A, Isabel C. Morbilidad sentida de las urgencias médicas y la utilización de los servicios de salud en Medellín, Colombia, 2005-2006. Biomédica. 2012:180-9.

8. Oliveira GN, de Freitas Neves M, Muglia Araujo IE, Carvalho Filho MA. Profile of the population cared for in a referral emergency unit. Rev. Latino-Am. Enfermagem. 2011 May-Jun;19(3):548-56.

9. Ortega Maján MT, Rabanaque Hernández MJ, Júdez Legaristi D, Cano del Pozo MI, Abad Díez JM, Moliner Lahoz J. Perfil de los usuarios y motivos de demanda del servicio de urgencias extrahospitalario 061. Emergencias. 2008;20:27-34.

10. Braun T, García Castrillo-Riesgo L, Krafft T, Díaz-Regañón Vilches G. Use of emergency medical service and sociodemographic factors. Gac Sanit. 2002 MarApr;16(2):139-44

11. Colombia. Ministerio de la Protección Social. Biblioteca Nacional de Indicadores de Calidad de laAtención en Salud, actualización: Junio 2011. Bogotá: El Ministerio; 2012. 